\title{
The Folding Deuteron Optical Model Potentials
}

\author{
Xiaohua Li.* Haixia An, and Chonghai Ca $\overbrace{}^{+}$ \\ Department of Physics, Nankai University, Tianjin, China 300071 Tel:86-22-23494154
}

For 52 target nuclei with deuteron as projectile, we calculate the reaction cross sections and elastic scattering angular distributions, as well as the $\chi^{2}$ values for 11 kinds of deuteron optical model potentials: our global deuteron optical potentials and 10 folding optical potentials calculated with 2 phenomenological global nucleon optical potentials given by Koning et al(KD) and by Varneret al(CH89), and 8 microscopic nucleon optical potentials with the generalized Skyrme force parameters(GS1-6) and modified Skyrme force parameters(SKa, SKb).

We find that for constructing the folding deuteron optical potential, both SKa and SKb are the best Skyrme force parameters of the microscopic nucleon optical potential proposed by Q. Shen et al.

\section{INTRODUCTION}

Deuteron is the simplest compound particle consisting of a proton and a neutron. It was suggested[1] [2] [3] that, to the first approximation, the optical potential of a compound particle should be the sum of the optical potentials of its constituent nucleons, suitably averaged over their internal density distributions in the compound particle.

Recently, H. An and C. Cai[4] obtained a global deuteron optical model potential for almost all target nuclei ranging from ${ }^{12} \mathrm{C}$ to ${ }^{238} \mathrm{U}$ in the energy region below $183 \mathrm{MeV}$; we call it GL. It is a phenomenological optical potential containing many adjustable parameters and can reproduce the existing experimental data well. However, for those nuclei that lack experimental data, especially for the nuclei that are far from the beta-stability line, we can not with full confidence use it to predict the reaction cross sections and elastic scattering angular distributions, because it has no solid theoretical basis.

In this work, we calculate the deuteron optical model potential by folding the proton and neutron optical potential obtained with the phenomenological method and one of the microscopic approaches proposed by Q. Shen et al [5] which

\footnotetext{
*Electronic address: lixiaohua@mail.nankai.edu.cn

†Electronic address: haicai@nankai.edu.cn
} 
has analytical formula and is suit for a large amount of calculations in nuclear data evaluation and analysis, and all of them do not contain any free parameters in our calculation of deuteron optical potentials, the reaction cross sections and the angular distributions of elastic scattering. The $\chi^{2}$ represents the degree of agreement between the calculated values of the reaction and differential elastic cross sections and their experimental data. We compare the $\chi^{2}$ values calculated with our global deuteron optical potential(GL) and with ten kinds of folding deuteron optical potentials, which correspond to ten kinds of nucleon optical potentials: the phenomenological global optical potential given by Koning et al $[6](\mathrm{KD})$ and by Varner et al [7] $(\mathrm{CH} 89)$ and the microscopic optical potential [5] with the generalized Skyrme force parameters (GS1-6)[8], and modified Skyrme force parameters (SKa, SKb) [9] for many nuclei. If we use the folding deuteron optical potential which are obtained from microscopic nucleon optical potential to calculate the reaction and differential elastic cross sections for those nuclei with plenty of experimental data, and obtain good theoretical results in agreement with experimental values, we can with confidence extend it to those nuclei which lack experimental data, because it has a reliable theoretical basis. Q. Shen and co-works made a large amount of calculations of neutron cross sections and angular distributions for various nuclei and obtained rather good results in accordance with experimental data $[10]-[15]$. They found that for neutron as projectile, the Skyrme force parameter GS2 is the best one and the next is SKa. In this work we will find which is the best Skyrme parameter for deuteron as projectile.

This paper is arranged as follows. In Sec. II, we provide the folding model and deuteron ground state wave function used in folding calculation. Sec. III gives results and discussion. Finally, a summary is given in Sec. IV.

\section{THE FOLDING MODEL AND DEUTERON GROUND STATE WAVE FUNCTION}

\section{A. The folding model}

The folding model used for the deuteron optical potential $U_{d}$ is

$$
\begin{aligned}
U_{d}(\vec{r}) & =<U_{n}+U_{p}+V_{c}>+\lambda_{\pi}^{2} \frac{V_{s o}+i W_{s o}}{r} \frac{d f_{s o}(r)}{d r} 2 \vec{S} \cdot \vec{l} \\
& =\int d \vec{s}\left[U_{n}\left(\overrightarrow{r_{n}}\right)+U_{p}\left(\overrightarrow{r_{p}}\right)+V_{c}\left(\overrightarrow{r_{p}}\right)\right] \phi_{d}^{2}(\vec{s})+\lambda_{\pi}^{2} \frac{V_{s o}+i W_{s o}}{r} \frac{d f_{s o}(r)}{d r} 2 \vec{S} \cdot \vec{l}
\end{aligned}
$$

where

$$
f_{\text {so }}(r)=\left[1+\exp \left(\left(r-r_{\text {so }} A^{1 / 3}\right) / a_{s o}\right)\right]^{-1}
$$


Here $U_{n}$ and $U_{p}$ are, respectively, the neutron and proton optical potentials containing central real part, surface absorption and volume absorption imaginary part, at half the bombarding energy of the deuteron. $V_{\text {so }}$ and $W_{\text {so }}$ are, respectively, the real and imaginary part of spin-orbital coupling potential of deuteron. $\lambda_{\pi}$ is the Compton wave length of pion, usually $\lambda_{\pi}^{2}=2.0 \mathrm{fm}^{2} . V_{c}(r)$ is the coulomb potential. That is to say, in this work, the folding model is only used for central real, imaginary part and coulomb potentials. The spin-orbital coupling potential can not be treated as the central potential in folding model because the spin is $1 / 2$ for neutron and proton and 1 for deuteron. Then we suppose that the spin-orbital coupling potential as well as their parameters are the same as in Ref. [4]. And $\phi_{d}$ is the deuteron ground state wave function used in folding integration. In the work of F. G. Perey and G. R. Satchler [16], they chose Hulthén function as the deuteron ground state wave function. In this work, we calculate it with numerical method, which will be discussed in the following section. Here $\vec{s}=\overrightarrow{r_{n}}-\overrightarrow{r_{p}}$, and $\vec{r}=\frac{1}{2}\left(\overrightarrow{r_{n}}+\overrightarrow{r_{p}}\right)$ is the position of the center of mass of the deuteron.

\section{B. The deuteron ground state wave function}

The deuteron consists of a proton and a neutron with a bing energy of $\varepsilon_{0}=2.2259 \mathrm{MeV}$. The Hamiltonian of the deuteron system can be written as:

$$
H=\frac{p^{2}}{2 m}+V(r)
$$

where $\mathrm{V}(\mathrm{r})$ is the interaction between proton and neutron in deuteron and $\mathrm{m}$ is the reduced mass of the deuteron system. We choose $\mathrm{V}(\mathrm{r})$ as the Gauss form $[17]$

$$
V(r)=-v_{0} \exp \left[-\left(\frac{r}{r_{0}}\right)^{2}\right]
$$

In Ref.[17], the parameters of $v_{0}$ and $r_{0}$ are taken as $72.15 \mathrm{MeV}$ and $1.484 \mathrm{fm}$, respectively. The eigen equation of the deuteron ground state can be written as

$$
\left(H-\varepsilon_{0}\right) \phi_{d}(\vec{r})=0 \quad \text { with } \quad \varepsilon_{0}=2.2259 \mathrm{MeV}
$$

where $\phi_{d}(\vec{r})$ is the deuteron ground state function. We solve this equation with numerical method[18]. In order to fit the experimental data of deuteron bound energy $\varepsilon_{0}=2.2259 \mathrm{MeV}$, we change the parameter $v_{0}$ as $72.194 \mathrm{MeV}$, which is a little different from that in Ref.[17]. The wave function of the deuteron ground state varying with $r$ is plotted in Fig 1. 


\section{RESULTS AND DISCUSSION}

Our theoretical calculation is carried out in the non-relativistic frame, no consideration is given to the relativistic kinetics corrections because they are usually very small when $E \leq 200 \mathrm{MeV}$ (see Ref. [4]). All experimental data used in this work are taken from EXFOR(web address: http://www.nndc.bnl.gov/). As for data errors, usually we take the values given in EXFOR; in the case that the data errors are not provided in EXFOR, we take them as $10 \%$ of the corresponding experimental data.

For a certain nuclide, the $\chi^{2}$ represents the deviation of the calculated values from the experimental data, which is defined as follows:

$$
\chi^{2}=\frac{\frac{W_{n o n}}{N_{n o n}} \sum_{i=1}^{N_{n o n}}\left(\frac{\sigma_{n o n, i}^{t h}-\sigma_{n o n, i}^{e x p}}{\Delta \sigma_{n o n, i}^{e x p}}\right)^{2}+\frac{W_{e l}}{N_{e l}} \sum_{i=1}^{N_{e l}} \frac{1}{N_{i}} \sum_{j=1}^{N_{i}}\left(\frac{\sigma_{e l}^{t h}(i, j)-\sigma_{e l}^{e x p}(i, j)}{\Delta \sigma_{e l}^{e x p}(i, j)}\right)^{2}}{W_{n o n}+W_{e l}}
$$

where $\sigma_{e l}^{t h}(i, j)$ and $\sigma_{e l}^{e x p}(i, j)$ are the theoretical and experimental differential cross sections at the j-th angle with the i-th incidence energy, respectively. The subscript el means the data are for the elastic scattering angular distribution. $\Delta \sigma_{e l}^{e x p}(i, j)$ is the error of corresponding experimental data. $N_{i}$ is the number of angles for the i-th incidence energy. $N_{e l}$ is the number of incident energy points of elastic scattering angular distribution for a given target nucleus. $\sigma_{n o n}^{t h}, i$ and $\sigma_{n o n, i}^{\text {exp }}$ are the theoretical and experimental reaction cross sections at the i-th incidence energy, respectively. The subscript non means the data are for the reaction (or nonelastic) cross sections. $\Delta \sigma_{n o n}^{\text {exp }} i$ is the error of corresponding experimental data. $N_{\text {non }}$ is the number of incident energy points of reaction cross sections for a given target nucleus. The $W_{e l}$ and $W_{n o n}$ are the weight of angular distribution of elastic scattering and reaction cross sections, respectively. As for the weights of $W_{e l}$ and $W_{n o n}$, we believe that experimental data of all nuclei are same reliable, and equal weights are applied with $W_{e l}=2.0$ and $W_{n o n}=0.1$ as in Ref. [4] $]$.

The target nuclei and the corresponding incident energies used in calculations are showed in Table I. And we use $\chi_{n G L}^{2}$ to express the $\chi^{2}$ calculated with the global deuteron optical potential 4$]$ for the $\mathrm{n}$-th nucleus, the $\chi_{n i}^{2}$ for $\mathrm{i}=\mathrm{KD}$, CH89, GS1-6, SKa, SKb(see Table II-V) to express the $\chi^{2}$ calculated with the folding deuteron optical potential (see Eq.(1)) using the nucleon optical potential parameters of Koning et al [6] , Varner et al [7], microscopic optical potential parameters [8] 9], respectively. The results are given in Table II-V. As for calculating the microscopic optical potential of nucleon, we use the analytical formula given by Q. Shen et al [5]].

In order to choose the best one for constructing folding deuteron optical potentia among the ten kinds of nucleon 
optical potentials, we define

$$
\begin{aligned}
& \overline{\chi_{i}^{2}}=\frac{1}{N} \sum_{n=1}^{N} \chi_{n i}^{2} \quad \text { with } \quad i=G L, K D, C H 89, S K a, S K b, G S 1-6 \\
& \overline{\chi_{n i}^{2}}=\frac{11 \chi_{n i}^{2}}{\sum_{j} \chi_{n j}^{2}} \quad \text { with } \quad i, j=G L, K D, C H 89, S K a, S K b, G S 1-6 \\
& <\overline{\chi_{i}^{2}}>=\frac{1}{N} \sum_{n=1}^{N} \chi_{n i}^{\overline{2}} \quad \text { with } \quad i=G L, K D, C H 89, S K a, S K b, G S 1-6
\end{aligned}
$$

where $\mathrm{N}=52$ is the total number of nuclei in Table I. For a given target nucleus n, the $\chi_{n i}^{\overline{2}}$ defined in eq. (8) is the relative $\chi^{2}$ of i-th optical potential among the 11 kinds of optical potentials. For these 52 nuclei, $\overline{\chi_{i}^{2}}$ defined in eq. (7) is the average value of these $\chi^{2}$ themselves; $\left\langle\overline{\chi_{i}^{2}}\right\rangle$ defined in eq. (9) is the average value of the relative $\chi^{2}$, it can express more clearly than $\overline{\chi_{i}^{2}}$ which is the best one among the 10 kinds of nucleon optical potentials. The values of these $\chi_{n i}^{2}$ and $\bar{\chi}_{n i}^{2}$ are given in Table II-V, and both the $\overline{\chi_{i}^{2}}$ and $\left\langle\bar{\chi}^{2}{ }_{i}>\right.$ are given in Table VI.

From Table II-V, we can see that except for ${ }^{6} \mathrm{Li},{ }^{9} \mathrm{Be},{ }^{11} \mathrm{~B},{ }^{14} \mathrm{~N},{ }^{27} \mathrm{Al},{ }^{112} \mathrm{Sn}$, the value of $\chi_{n G L}^{2}$ is always the minimum one among the $11 \chi_{n i}^{2}$ values because the parameters in global deuteron optical potential are obtained based on the experimental data of total reaction cross sections and elastic scattering angular distributions of deuteron itself. Generally speaking, the $\chi_{n i}^{2}$ for $\mathrm{i}=\mathrm{Ch} 89, \mathrm{KD}, \mathrm{SKa}$, SKb, are with small values and closed to each other for all the 52 nuclei, and the values of $\chi_{n i}^{2}$ for $\mathrm{i}=$ GS1-6 are much larger than those of Ch89, KD, SKa, SKb for most of the 52 nuclei. From Table VI we can clearly see that for both $\overline{\chi_{i}^{2}}$ and $<\bar{\chi}^{2}{ }_{i}>$, SKa and SKb are with nearly equal small values which are less than those of KD and CH89, and the values of GS1-6 are much larger than those of SKa, SKb, KD and CH89. Therefore, we can conclude that the microscopic nucleon optical potential with the generalized Skyrme force parameters(GS1-6) 8] are not suitable for constructing folding deuteron optical potential. And SKa and SKb are two best Skyrme force parameters of microscopic nucleon optical potentials for constructing folding deuteron optical potential.

For giving an intuitional display, as examples we plot the experimental data and the theoretical values of the elastic scattering angular distributions for the targets ${ }^{24} \mathrm{Mg}$ and ${ }^{120} \mathrm{Sn}$ in Figs. 2, 3, respectively; and the reaction cross sections for the targets ${ }^{27} \mathrm{Al},{ }^{90} \mathrm{Zr},{ }^{120} \mathrm{Sn}$ and ${ }^{208} \mathrm{~Pb}$ in Fig. 4. The theoretical values are calculated for the global deuteron optical potential [4] and two sets of the folding deuteron optical potentials obtained from the phenomenological global nucleon optical potential given by Varner et al 7] (CH89) and the microscopic optical potential with modified Skyrme force parameters SKa $[9]$. From Figs. 2-4 we can see that the calculated values for two sets of folding optical 
potential can basically reproduce the experimental data. In Figs. 5, 6 we also give the real part and imaginary part of three sets of deuteron optical potentials at the incident energies 1, 10, 100, $200 \mathrm{MeV}$ for target ${ }^{208} \mathrm{~Pb}$. Among the three sets of deuteron optical potentials, there are two sets of folding optical potentials constructed with the phenomenological global nucleon optical potential CH89 and the microscopic nucleon optical potential with the modified Skyrme force parameters SKa, as well as the global deuteron optical potential(GL) [4]. In all figures, the solid lines represent the values calculated with GL, the dashed lines correspond to CH89, and the dotted lines to SKa. To see clearly, in Fig. 5, for the real part of deuteron optical potentials, the curves at the top represent the true values, while the others are multiplied by a factor of 2,3 and 4 , respectively; in Fig. 6 , for the imaginary part, the curves at the top represent true values, while the others are multiplied by a factor of 10,15 and 20, respectively.

\section{SUMMARY}

In this work, for 52 target nuclei with deuteron as projectile, we compare the $\chi_{n i}^{2}, \chi_{n i}^{\overline{2}}, \overline{\chi_{i}^{2}}$ and $<\overline{\chi^{2}}{ }_{i}>$ values for 11 kinds of deuteron optical model potentials: the global deuteron optical potential [4] and 10 folding optical potentials calculated with 2 phenomenological global nucleon optical potentials given by Koning et al(KD) and by Varneret al(CH89), and 8 microscopic nucleon optical potentials [5] with the generalized Skyrme force parameters(GS1-6) and the modified Skyrme force parameters(SKa, SKb).

For giving an intuitional display, we plot the experimental data and the theoretical values of the elastic scattering angular distributions and/or the reaction cross sections of the targets ${ }^{24} \mathrm{Mg},{ }^{27} \mathrm{Al},{ }^{90} \mathrm{Zr},{ }^{120} \mathrm{Sn}$ and ${ }^{208} \mathrm{~Pb}$ for three kinds of deuteron optical potentials. We also compare the same three kinds of deuteron optical potential themselves for target ${ }^{208} \mathrm{~Pb}$.

Through the comparisons, we find that for constructing folding deuteron optical potential, the microscopic nucleon optical potential[5] with Skyrme force parameters SKa and SKb are the best two because they can reproduce the experimental data some better than the phenomenological global nucleon optical potentials: KD and CH89. Considering that for neutron as projectile, the microscopic nucleon optical potential Skyrme force parameters GS2 and Ska are the best two, we conclude that Ska is the best Skyrme force parameter of microscopic nucleon optical potential for both neutron and deuteron as projectiles. The microscopic nucleon optical potential has reliable theoretical basis, we can with confidence use it to predict the data of cross sections and angular distributions for those nuclei which lack experimental data and are outside the applicable ranges of the global deuteron optical potential[4]. 


\section{References}

[1] S. Watanabe, Nucl. Phys. 8 (1958) 484.

[2] G. R. Satchler, Nucl. Phys. 21 (1960) 116.

[3] J. R. Rook, Nucl. Phys. 61 (1965) 219.

[4] H. An, C. Cai, Phys. Rev. C, 73 (2006) 054605.

[5] Q. Shen, J. Zhang, Y. Tian, Z. Ma, Y. Zhuo, Z. Phys. A 303 (1981) 69.

[6] A. J. Koning, J. P. Delaroche, Nucl. Phys. A 713 (2003) 231.

[7] R. L. Varner, W. J. Thompson, T. L. Mcabee, E. J. Ludwig, T. B. Clegg, Phys. Rep. 201 (1991) 57.

[8] S. Krewald, V. Klemt, A. Faessler, Nucl. Phys. A 281 (1977) 166.

[9] H. S. Kohler, Nucl. Phys. A 258 (1976) 301.

[10] C. Cai, Q. Shen, Y. Zhuo, Nucl. Sci. Eng. 109 (1991) 142.

[11] Y. Tian et al, Chinese J. Nucl. Phys. 7 (1985) 154.

[12] Y. Tian et al, Chinese J. Nucl. Phys. 7 (1985) 207.

[13] Y. Tian et al, Chinese J. Nucl. Phys. 7 (1985) 344.

[14] Y. Tian et al, Chinese J. Nucl. Phys. 8 (1986) 28.

[15] Y. Tian et al, Chinese J. Nucl. Phys. 10 (1988) 183.

[16] F. G. Perey, G. R. Satchler, Nucl. Phys. A 97 (1967) 515.

[17] N. Austern, Y. Iseri, M. Kamimura, M. Kawai, G. Rawitscher, M. Yahiro, Phys. Rep. 154 (1987) 125.

[18] C. Cai, L. Li, Chinese J. High Ener. Phys. \& Nucl. Phys. 27 (2003) 1005.

[19] K. Hatanaka, K. Imai, S. Kobayashi, T. Matsusue, M. Nakamura, K. Nisimura, T. Noro, H. Sakamoto, H. Shimizu, J. Shirai, Nucl. Phys. A 340 (1980) 93.

[20] A. Kiss, O. Aspelund, G. Hrehuss, K. T. Knöpfle, M. Rogge, U. Schwinn, Z. Seres, P. Turek, C. Mayer-Böricke, Nucl. Phys. A 262 (1976) 1.

[21] C. Bäumer, R. Bassini, A. M. van den Berg, D. De Frenne, D. Frekers, M. Hagemann, V. M. Hannen, M. N. Harakeh, J. Heyse, M. A. de Huu, E. Jacobs, M. Mielke, S. Rakers, R. Schmidt, H. Sohlbach, H. J. Wörtche, Phys. Rev. C 63 (2001) 037601.

[22] J. M. Lohr, W. Haeberli, Nucl. Phys. A 232 (1974) 381.

[23] R. K. Jolly, E. K. Lin, B. L. Cohen, Phys. Rev. 130 (1963) 2391.

[24] M. Ermer, H. Clement, G. Holetzke, W. Kabitzke, G. Graw, R. Hertenberger, H. Kader, F. Merz, P. Schiemenz , Nucl. Phys. A 533 (1991) 71. 
[25] G. Perrin, Nguyen van Sen, J. Arvieux, C. Perrin, R. Darves-Blanc, J. L. Durand, A. Fiore, J. C. Gondrand, F. Merchez. Nucl. Phys. A 206 (1973) 623.

[26] J. Bojowald, H. Machner, H. Nann, W. Oelert, M. Rogge, P. Turek, Phys. Rev. C 38 (1988) 1153.

[27] G. Duhamel, L. Marcus, H. Langevin-Joliot, J. P. Didelez, P. Narboni, C. Stephan, Nucl. Phys. A 174 (1971) 485.

[28] S. Mayo, W. Schimmerling, M. J. Sametband, R. M. Eisberg, Nucl. Phys. 62 (1965) 393.

[29] J. R. Wu, C. C. Chang, H. D. Holmgren, Phys. Rev. C 19 (1979) 370.

[30] G. P. Millburn, W. Birnbaum, W. E. Crandall, L. Schecter, Phys. Rev. 95 (1954) 1268.

[31] A. Auce, R. F. Carlson, A. J. Cox, A. Ingemarsson, R. Johansson, P. U. Renberg, O. Sundberg, G. Tibell, Phys. Rev. C 53 (1996) 2919. 
TABLE I: Incident deuteron energy for every nuclide used in the calculations

\begin{tabular}{|c|c|c|c|c|c|}
\hline Nucleus & $\begin{array}{c}\text { Number of } \\
\text { energy points }\end{array}$ & energy $(\mathrm{MeV})$ & Nucleus & $\begin{array}{c}\text { Number of } \\
\text { energy points }\end{array}$ & energy $(\mathrm{MeV})$ \\
\hline${ }^{6} \mathrm{Li}$ & 25 & $3-27.3$ & ${ }^{59} \mathrm{Co}$ & 1 & 6.5 \\
\hline${ }^{9} \mathrm{Be}$ & 35 & $1.1-160$ & ${ }^{58} \mathrm{Ni}$ & 13 & $10.98-120$ \\
\hline${ }^{10} \mathrm{~B}$ & 1 & 11.8 & ${ }^{60} \mathrm{Ni}$ & 14 & 8.9-97.4 \\
\hline${ }^{11} \mathrm{~B}$ & 2 & $5.5-11.8$ & ${ }^{65} \mathrm{Cu}$ & 3 & $11-34.4$ \\
\hline${ }^{12} \mathrm{C}$ & 16 & $4-170$ & ${ }^{68} \mathrm{Zn}$ & 8 & $9-80$ \\
\hline${ }^{13} \mathrm{C}$ & 2 & $13.7-17.7$ & ${ }^{70} \mathrm{Ge}$ & 1 & 171 \\
\hline${ }^{14} \mathrm{~N}$ & 12 & $1.3-52$ & ${ }^{72} \mathrm{Ge}$ & 1 & 171 \\
\hline${ }^{16} \mathrm{O}$ & 19 & $0.98-171$ & ${ }^{74} \mathrm{Ge}$ & 1 & 6.02 \\
\hline${ }^{20} \mathrm{Ne}$ & 1 & 52 & ${ }^{86} \mathrm{Sr}$ & 1 & 88 \\
\hline${ }^{22} \mathrm{Ne}$ & 1 & 52 & ${ }^{89} \mathrm{Y}$ & 6 & $15-85$ \\
\hline${ }^{24} \mathrm{Mg}$ & 17 & $52-170$ & ${ }^{90} \mathrm{Zr}$ & 13 & $5.5-183$ \\
\hline${ }^{27} \mathrm{Al}$ & 17 & $5-160$ & ${ }^{93} \mathrm{Nb}$ & 5 & $11.8-52$ \\
\hline${ }^{28} \mathrm{Si}$ & 18 & $1.1-97.4$ & ${ }^{103} \mathrm{Rh}$ & 5 & $11.8-52$ \\
\hline${ }^{32} \mathrm{~S}$ & 5 & $11.8-171$ & ${ }^{112} \mathrm{Sn}$ & 1 & 23 \\
\hline${ }^{40} \mathrm{Ar}$ & 3 & $11.8-56$ & ${ }^{116} \mathrm{Sn}$ & 6 & $23-183$ \\
\hline${ }^{39} \mathrm{~K}$ & 1 & 12.8 & ${ }^{118} \mathrm{Sn}$ & 3 & $14.5-56$ \\
\hline${ }^{40} \mathrm{Ca}$ & 10 & $5-140$ & ${ }^{120} \mathrm{Sn}$ & 12 & $11-97.4$ \\
\hline${ }^{44} \mathrm{Ca}$ & 4 & $9-56$ & ${ }^{124} \mathrm{Sn}$ & 5 & $14.5-97.4$ \\
\hline${ }^{48} \mathrm{Ca}$ & 7 & $9-97.4$ & ${ }^{140} \mathrm{Ce}$ & 1 & 52 \\
\hline${ }^{48} \mathrm{Ti}$ & 6 & $9-52$ & ${ }^{181} \mathrm{Ta}$ & 3 & $11.8-52$ \\
\hline${ }^{50} \mathrm{Ti}$ & 4 & $11.8-52$ & ${ }^{186} \mathrm{~W}$ & 1 & 12 \\
\hline${ }^{50} \mathrm{~V}$ & 1 & 171 & ${ }^{197} \mathrm{Au}$ & 6 & $11-52$ \\
\hline${ }^{51} \mathrm{~V}$ & 4 & $13.6-171$ & ${ }^{206} \mathrm{~Pb}$ & 2 & $11.8-79.4$ \\
\hline${ }^{52} \mathrm{Cr}$ & 11 & $4.39-34.4$ & ${ }^{208} \mathrm{~Pb}$ & 29 & $9-140$ \\
\hline${ }^{54} \mathrm{Fe}$ & 9 & $10-56$ & ${ }^{209} \mathrm{Bi}$ & 3 & $11-52$ \\
\hline${ }^{56} \mathrm{Fe}$ & 8 & $5-56$ & ${ }^{232} \mathrm{Th}$ & 3 & $11.8-70$ \\
\hline
\end{tabular}


TABLE II: $\chi_{n i}^{2}$ and $\chi_{n i}^{\overline{2}}$ for every nuclide

\begin{tabular}{|c|c|c|c|c|c|c|c|c|c|c|c|}
\hline Nucleus & $\begin{array}{l}\chi_{n G L}^{2} \\
\bar{\chi}_{n G L}^{2}\end{array}$ & $\begin{array}{l}\chi_{n C H 89}^{2} \\
\overline{\chi_{n C H 89}^{2}}\end{array}$ & $\begin{array}{l}\chi_{n K D}^{2} \\
\overline{\chi_{n K D}^{2}}\end{array}$ & $\begin{array}{l}\chi_{n S K a}^{2} \\
\bar{\chi}_{n S K a}^{2}\end{array}$ & $\begin{array}{l}\chi_{n S K b}^{2} \\
\bar{\chi}_{n S K b}^{2}\end{array}$ & $\begin{array}{l}\chi_{n G S 1}^{2} \\
\bar{\chi}_{n G S 1}^{2}\end{array}$ & $\begin{array}{l}\chi_{n G S 2}^{2} \\
\overline{\chi^{2}} \\
n G S 2\end{array}$ & $\begin{array}{l}\chi_{n G S 3}^{2} \\
\overline{\chi^{2}}{ }_{n G S 3}\end{array}$ & $\begin{array}{l}\chi_{n G S 4}^{2} \\
\bar{\chi}_{n G S 4}^{2}\end{array}$ & $\begin{array}{l}\chi_{n G S 5}^{2} \\
\bar{\chi}_{n G S 5}^{2}\end{array}$ & $\begin{array}{l}\chi_{n G S 6}^{2} \\
\overline{\chi^{2}}{ }_{n G S 6}\end{array}$ \\
\hline \multirow[t]{2}{*}{${ }^{6} \mathrm{Li}$} & 192.79 & 180.93 & 191.76 & 214.60 & 213.63 & 214.18 & 219.82 & 229.01 & 229.31 & 240.42 & 255.39 \\
\hline & 0.89035 & 0.8356 & 0.88561 & 0.99106 & 0.98661 & 0.98914 & 1.01518 & 1.05763 & 1.05903 & 1.11031 & 1.17948 \\
\hline \multirow[t]{2}{*}{${ }^{9} \mathrm{Be}$} & 347.55 & 324.29 & 401.32 & 358.08 & 394.70 & 788.80 & 782.92 & 586.44 & 396.64 & 385.59 & 296.67 \\
\hline & 0.80266 & 0.74893 & 0.92683 & 0.82698 & 0.91155 & 1.8217 & 1.80813 & 1.35437 & 0.91603 & 0.8905 & 0.68516 \\
\hline \multirow[t]{2}{*}{${ }^{10} \mathrm{~B}$} & 53.007 & 189.49 & 189.49 & 189.49 & 189.49 & 189.49 & 189.49 & 189.49 & 189.50 & 189.50 & 189.50 \\
\hline & 0.29933 & 1.07004 & 1.07004 & 1.07006 & 1.07006 & 1.07005 & 1.07005 & 1.07006 & 1.0701 & 1.07009 & 1.07011 \\
\hline \multirow[t]{2}{*}{${ }^{11} \mathrm{~B}$} & 66.732 & 57.876 & 85.122 & 66.676 & 66.618 & 98.206 & 111.54 & 127.37 & 73.710 & 79.773 & 108.50 \\
\hline & 0.77914 & 0.67575 & 0.99386 & 0.7785 & 0.77781 & 1.14663 & 1.30235 & 1.48709 & 0.86062 & 0.93141 & 1.26683 \\
\hline \multirow[t]{2}{*}{${ }^{12} \mathrm{C}$} & 98.069 & 165.71 & 100.25 & 148.18 & 145.35 & 183.99 & 170.60 & 162.88 & 503.48 & 373.56 & 344.33 \\
\hline & 0.45016 & 0.76064 & 0.46017 & 0.68017 & 0.66718 & 0.84456 & 0.78309 & 0.74765 & 2.31108 & 1.71471 & 1.58058 \\
\hline \multirow[t]{2}{*}{${ }^{13} \mathrm{C}$} & 103.93 & 416.02 & 416.01 & 416.01 & 416.01 & 416.01 & 416.01 & 416.01 & 415.92 & 415.92 & 415.91 \\
\hline & 0.26811 & 1.07328 & 1.07325 & 1.07324 & 1.07326 & 1.07326 & 1.07327 & 1.07326 & 1.07302 & 1.07303 & 1.07301 \\
\hline \multirow[t]{2}{*}{${ }^{14} \mathrm{~N}$} & 80.641 & 87.556 & 71.851 & 78.696 & 72.340 & 49.512 & 53.878 & 79.811 & 116.90 & 117.53 & 142.36 \\
\hline & 0.93268 & 1.01266 & 0.83101 & 0.91018 & 0.83668 & 0.57265 & 0.62314 & 0.92308 & 1.35202 & 1.35937 & 1.64653 \\
\hline \multirow[t]{2}{*}{${ }^{16} \mathrm{O}$} & 59.762 & 82.545 & 77.150 & 144.67 & 143.76 & 210.70 & 239.24 & 275.10 & 263.72 & 300.52 & 305.93 \\
\hline & 0.31258 & 0.43174 & 0.40352 & 0.75667 & 0.75193 & 1.10202 & 1.25134 & 1.43886 & 1.37939 & 1.57182 & 1.60012 \\
\hline \multirow[t]{2}{*}{${ }^{20} \mathrm{Ne}$} & 256.80 & 923.06 & 571.15 & 368.48 & 361.51 & 1852.2 & 2050.9 & 1882.2 & 1480.0 & 1565.4 & 1279.7 \\
\hline & 0.22434 & 0.8064 & 0.49897 & 0.32191 & 0.31582 & 1.61813 & 1.79166 & 1.64429 & 1.29297 & 1.3676 & 1.11793 \\
\hline \multirow[t]{2}{*}{${ }^{22} \mathrm{Ne}$} & 174.01 & 501.73 & 460.37 & 264.27 & 263.75 & 913.28 & 1109.0 & 1130.3 & 616.47 & 717.67 & 776.51 \\
\hline & 0.27631 & 0.79671 & 0.73102 & 0.41964 & 0.41881 & 1.45021 & 1.76104 & 1.79475 & 0.97889 & 1.1396 & 1.23302 \\
\hline \multirow[t]{2}{*}{${ }^{24} \mathrm{Mg}$} & 17.392 & 195.36 & 59.556 & 31.376 & 27.714 & 199.57 & 197.11 & 169.11 & 629.08 & 505.87 & 233.82 \\
\hline & 0.08443 & 0.94838 & 0.28912 & 0.15231 & 0.13453 & 0.96879 & 0.95686 & 0.82095 & 3.05384 & 2.45574 & 1.13506 \\
\hline \multirow[t]{2}{*}{${ }^{27} \mathrm{Al}$} & 88.976 & 137.92 & 79.191 & 179.09 & 191.32 & 1623.6 & 1986.4 & 546.51 & 281.54 & 302.95 & 301.30 \\
\hline & 0.17114 & 0.26529 & 0.15232 & 0.34447 & 0.368 & 3.12289 & 3.82086 & 1.05121 & 0.54154 & 0.58273 & 0.57954 \\
\hline \multirow[t]{2}{*}{${ }^{28} \mathrm{Si}$} & 31.529 & 44.752 & 58.603 & 93.905 & 99.431 & 483.77 & 590.94 & 273.99 & 175.02 & 211.54 & 129.23 \\
\hline & 0.15817 & 0.2245 & 0.29399 & 0.47108 & 0.49881 & 2.42691 & 2.9645 & 1.37451 & 0.878 & 1.0612 & 0.64831 \\
\hline
\end{tabular}


TABLE III: $\chi_{n i}^{2}$ and $\chi_{n i}^{\overline{2}}$ for every nuclide

\begin{tabular}{|c|c|c|c|c|c|c|c|c|c|c|c|}
\hline leus & $\begin{array}{l}\chi_{n G L}^{2} \\
\bar{\chi}_{n G L}^{2} \\
\end{array}$ & $\begin{array}{l}\chi_{n C H 89}^{2} \\
\bar{\chi}_{n C H 89}^{2}\end{array}$ & $\begin{array}{l}\chi_{n K D}^{2} \\
\bar{\chi}_{n K D}^{2}\end{array}$ & $\begin{array}{l}\chi_{n S K a}^{2} \\
\bar{\chi}_{n S K a}^{2}\end{array}$ & $\begin{array}{l}\chi_{n S K b}^{2} \\
\bar{\chi}_{n S K b}^{2}\end{array}$ & $\begin{array}{c}\chi_{n G S 1}^{2} \\
\bar{\chi}_{n G S 1}^{2}\end{array}$ & $\begin{array}{c}\chi_{n G S 2}^{2} \\
\bar{\chi}_{n G S 2}^{2}\end{array}$ & $\begin{array}{c}\chi_{n G S 3}^{2} \\
\bar{\chi}_{n G S 3}^{2}\end{array}$ & $\begin{array}{l}\chi_{n G S 4}^{2} \\
\bar{\chi}_{n G S 4}^{2}\end{array}$ & $\begin{array}{l}\chi_{n G S 5}^{2} \\
\bar{\chi}_{n G S 5}^{2} \\
\end{array}$ & $\begin{array}{l}\chi_{n G S 6}^{2} \\
\bar{\chi}_{n G S 6}^{2}\end{array}$ \\
\hline \multirow[t]{2}{*}{${ }^{32} \mathrm{~S}$} & 46.506 & 143.85 & 106.04 & 196.92 & 199.92 & 782.50 & 976.09 & 483.23 & 428.83 & 524.34 & 446.46 \\
\hline & 0.11802 & 0.36503 & 0.2691 & 0.49973 & 0.50732 & 1.98573 & 2.47699 & 1.22628 & 1.08822 & 1.3306 & 1.13297 \\
\hline \multirow{2}{*}{${ }^{40} \mathrm{Ar}$} & 10.236 & 123.82 & 190.36 & 298.92 & 321.71 & 1057.3 & 1425.6 & 1104.2 & 456.56 & 632.01 & 635.08 \\
\hline & 0.018 & 0.21771 & 0.33472 & 0.52561 & 0.56567 & 1.85916 & 2.50677 & 1.94159 & 0.80279 & 1.11129 & .11669 \\
\hline \multirow[t]{2}{*}{${ }^{39} \mathrm{~K}$} & 16.777 & 746.44 & 746.44 & 746.43 & 746.44 & 746.43 & 746.43 & 746.44 & 746.39 & 746.40 & 746.40 \\
\hline & 0.02467 & 1.09756 & 1.09756 & 1.09755 & 1.09755 & 1.09755 & 1.09755 & 1.09755 & 1.09748 & 1.09749 & 1.09749 \\
\hline \multirow[t]{2}{*}{${ }^{40} \mathrm{Ca}$} & 17.965 & 71.776 & 49.973 & 61.990 & 62.101 & 73.365 & 83.611 & 83.214 & 109.06 & 112.00 & 89.302 \\
\hline & 0.24266 & 0.96952 & 0.67501 & 0.83733 & 0.83884 & 0.99098 & 1.12939 & 1.12402 & 1.47313 & 1.51285 & 1.20626 \\
\hline \multirow[t]{2}{*}{${ }^{44} \mathrm{Ca}$} & 23.364 & 67.939 & 108.97 & 253.94 & 290.99 & 1230.0 & 1759.3 & 569.15 & 202.39 & 300.55 & 190.06 \\
\hline & 0.05143 & 0.14957 & 0.23989 & 0.55904 & 0.64062 & 2.70789 & 3.87297 & 1.25297 & 0.44555 & 0.66166 & 0.41841 \\
\hline \multirow[t]{2}{*}{${ }^{48} \mathrm{Ca}$} & 55.450 & 134.19 & 259.94 & 411.52 & 471.67 & 1228.9 & 1683.1 & 567.94 & 264.48 & 385.25 & 281.32 \\
\hline & 0.10619 & 0.25699 & 0.49781 & 0.78812 & 0.90331 & 2.35353 & 3.2233 & 1.08767 & 0.50651 & 0.7378 & 0.53877 \\
\hline \multirow[t]{2}{*}{${ }^{48} \mathrm{Ti}$} & 5.0079 & 29.856 & 73.242 & 135.59 & 159.81 & 558.35 & 783.93 & 248.39 & 47.289 & 78.595 & 39.342 \\
\hline & 0.02551 & 0.15209 & 0.37309 & 0.69071 & 0.81409 & 2.84422 & 3.99334 & 1.26529 & 0.24089 & 0.40037 & 0.20041 \\
\hline \multirow[t]{2}{*}{${ }^{50} \mathrm{Ti}$} & 30.358 & 212.42 & 73.78 & 1574.5 & 1871.1 & 137.7 & 9433.8 & 2633.1 & 418.30 & 943.98 & 321.91 \\
\hline & 0.01312 & 0.09181 & 0.37765 & 0.6805 & 0.80869 & 3.08495 & 4.07733 & 1.13804 & 0.18079 & 0.40799 & 0.13913 \\
\hline \multirow[t]{2}{*}{${ }^{50} \mathrm{~V}$} & 2.0380 & 120.37 & 25.741 & 8.0949 & 8.0943 & 13.712 & 13.745 & 11.603 & 72.738 & 91.814 & 25.518 \\
\hline & 0.05698 & 3.36512 & 0.71964 & 0.22631 & 0.22629 & 0.38334 & 0.38425 & 0.32437 & 2.0335 & 2.5668 & 0.71341 \\
\hline \multirow[t]{2}{*}{${ }^{51} \mathrm{~V}$} & 8.7021 & 72.869 & 272.48 & 264.24 & 305.01 & 1714.9 & 2400.2 & 503.78 & 227.03 & 361.30 & 234.37 \\
\hline & 0.01504 & 0.12593 & 0.47092 & 0.45667 & 0.52712 & 2.96376 & 4.14811 & 0.87065 & 0.39235 & 0.62441 & 0.40504 \\
\hline \multirow[t]{2}{*}{${ }^{52} \mathrm{Cr}$} & 18.344 & 106.05 & 106.05 & 106.05 & 106.05 & 106.05 & 106.05 & 106.05 & 106.02 & 106.03 & 106.03 \\
\hline & 0.18704 & 1.08139 & 1.08139 & 1.08136 & 1.08136 & 1.08134 & 1.08136 & 1.08136 & 1.08109 & 1.08116 & 1.08114 \\
\hline \multirow[t]{2}{*}{${ }^{54} \mathrm{Fe}$} & 7.6120 & 35.234 & 92.083 & 179.01 & 212.58 & 891.26 & 1298.1 & 358.16 & 81.396 & 135.69 & 90.474 \\
\hline & 0.02476 & 0.11461 & 0.29954 & 0.58231 & 0.6915 & 2.8992 & 4.22255 & 1.16506 & 0.26478 & 0.44138 & 0.2943 \\
\hline \multirow[t]{2}{*}{${ }^{56} \mathrm{Fe}$} & 70.286 & 246.58 & 1048.7 & 778.22 & 924.34 & 6484.5 & 9679.7 & 2476.7 & 512.00 & 894.62 & 481.51 \\
\hline & 0.03276 & 0.11495 & 0.48887 & 0.36277 & 0.43089 & 3.0228 & 4.51225 & 1.15455 & 0.23867 & 0.41703 & 0.22446 \\
\hline
\end{tabular}


TABLE IV: $\chi_{n i}^{2}$ and $\chi_{n i}^{\overline{2}}$ for every nuclide

\begin{tabular}{|c|c|c|c|c|c|c|c|c|c|c|c|}
\hline Nucleus & $\begin{array}{l}\chi_{n G L}^{2} \\
\bar{\chi}_{n G L}^{2}\end{array}$ & $\begin{array}{l}\chi_{n C H 89}^{2} \\
\bar{\chi}_{n C H 89}^{2}\end{array}$ & $\begin{array}{l}\chi_{n K D}^{2} \\
\bar{\chi}_{n K D}^{2}\end{array}$ & $\begin{array}{l}\chi_{n S K a}^{2} \\
\bar{\chi}_{n S K a}^{2}\end{array}$ & $\begin{array}{l}\chi_{n S K b}^{2} \\
\bar{\chi}_{n S K b}^{2}\end{array}$ & $\begin{array}{l}\chi_{n G S 1}^{2} \\
\bar{\chi}_{n G S 1}^{2}\end{array}$ & $\begin{array}{l}\chi_{n G S 2}^{2} \\
\bar{\chi}_{n G S 2}^{2}\end{array}$ & $\begin{array}{c}\chi_{n G S 3}^{2} \\
\bar{\chi}_{n G S 3}^{2}\end{array}$ & $\begin{array}{l}\chi_{n G S 4}^{2} \\
\bar{\chi}_{n G S 4}^{2}\end{array}$ & $\begin{array}{l}\chi_{n G S 5}^{2} \\
\bar{\chi}_{n G S 5}^{2}\end{array}$ & $\begin{array}{l}\chi_{n G S 6}^{2} \\
\overline{\chi^{2}}{ }_{n G S 6}\end{array}$ \\
\hline \multirow[t]{2}{*}{${ }^{59} \mathrm{Co}$} & 12.238 & 142.16 & 142.16 & 142.16 & 142.16 & 142.16 & 142.16 & 142.16 & 142.16 & 142.16 & 142.16 \\
\hline & 0.09388 & 1.09061 & 1.09061 & 1.09061 & 1.09061 & 1.09061 & 1.09061 & 1.09061 & 1.09061 & 1.09061 & 1.09061 \\
\hline \multirow[t]{2}{*}{${ }^{58} \mathrm{Ni}$} & 9.3105 & 127.99 & 688.65 & 318.95 & 348.17 & 2259.3 & 3407.3 & 781.33 & 1762.4 & 2231.7 & 555.31 \\
\hline & 0.0082 & 0.11272 & 0.60648 & 0.28089 & 0.30663 & 1.98974 & 3.0007 & 0.6881 & 1.55214 & 1.96537 & 0.48905 \\
\hline \multirow[t]{2}{*}{${ }^{60} \mathrm{Ni}$} & 14.074 & 193.92 & 277.88 & 223.37 & 258.05 & 753.79 & 1135.1 & 340.36 & 89.009 & 132.68 & 81.812 \\
\hline & 0.04423 & 0.60944 & 0.87332 & 0.70201 & 0.811 & 2.36901 & 3.56747 & 1.06967 & 0.27974 & 0.417 & 0.25712 \\
\hline \multirow[t]{2}{*}{${ }^{65} \mathrm{Cu}$} & 12.860 & 5751.0 & 5751.0 & 5750.9 & 5750.9 & 5750.9 & 5750.9 & 5750.9 & 5750.7 & 5750.8 & 5750.8 \\
\hline & 0.00246 & 1.09977 & 1.09977 & 1.09976 & 1.09976 & 1.09976 & 1.09976 & 1.09976 & 1.09972 & 1.09974 & 1.09973 \\
\hline \multirow[t]{2}{*}{${ }^{68} \mathrm{Zn}$} & 9.6331 & 56.503 & 348.34 & 344.70 & 421.49 & 2158.0 & 3523.2 & 838.89 & 127.42 & 251.82 & 85.743 \\
\hline & 0.01298 & 0.07611 & 0.46925 & 0.46435 & 0.56779 & 2.90698 & 4.74609 & 1.13007 & 0.17164 & 0.33923 & 0.1155 \\
\hline \multirow[t]{2}{*}{${ }^{70} \mathrm{Ge}$} & 6.4264 & 139.67 & 31.633 & 14.268 & 13.342 & 26.618 & 25.158 & 19.963 & 136.11 & 170.21 & 43.998 \\
\hline & 0.11267 & 2.44874 & 0.55462 & 0.25016 & 0.23392 & 0.46669 & 0.44109 & 0.35001 & 2.38633 & 2.98436 & 0.77142 \\
\hline \multirow[t]{2}{*}{${ }^{72} \mathrm{Ge}$} & 2.2557 & 419.81 & 121.76 & 17.184 & 13.203 & 75.478 & 75.501 & 44.475 & 351.02 & 439.51 & 92.181 \\
\hline & 0.01502 & 2.79472 & 0.81059 & 0.1144 & 0.08789 & 0.50246 & 0.50261 & 0.29607 & 2.33674 & 2.92584 & 0.61365 \\
\hline \multirow[t]{2}{*}{${ }^{74} \mathrm{Ge}$} & 31.177 & 335.37 & 335.37 & 335.37 & 335.37 & 335.37 & 335.37 & 335.37 & 335.37 & 335.37 & 335.37 \\
\hline & 0.10132 & 1.08987 & 1.08987 & 1.08987 & 1.08987 & 1.08987 & 1.08987 & 1.08987 & 1.08987 & 1.08987 & 1.08987 \\
\hline \multirow[t]{2}{*}{${ }^{86} \mathrm{Sr}$} & 32.495 & 572.13 & 771.57 & 53.665 & 54.013 & 218.95 & 182.90 & 94.659 & 117.53 & 110.62 & 80.547 \\
\hline & 0.15615 & 2.74933 & 3.7077 & 0.25788 & 0.25956 & 1.05215 & 0.87893 & 0.45488 & 0.56478 & 0.53158 & 0.38706 \\
\hline \multirow[t]{2}{*}{${ }^{89} \mathrm{Y}$} & 34.956 & 210.84 & 648.50 & 449.18 & 548.08 & 2410.4 & 4229.7 & 890.80 & 158.02 & 231.94 & 167.07 \\
\hline & 0.03853 & 0.2324 & 0.71481 & 0.49512 & 0.60412 & 2.65688 & 4.66225 & 0.9819 & 0.17417 & 0.25565 & 0.18416 \\
\hline \multirow[t]{2}{*}{${ }^{90} \mathrm{Zr}$} & 5.9884 & 55.942 & 210.10 & 209.68 & 228.67 & 441.86 & 620.39 & 200.14 & 113.85 & 164.23 & 120.96 \\
\hline & 0.02777 & 0.25945 & 0.9744 & 0.97247 & 1.06054 & 2.04924 & 2.87722 & 0.92822 & 0.52803 & 0.76168 & 0.56098 \\
\hline \multirow[t]{2}{*}{${ }^{93} \mathrm{Nb}$} & 6.0331 & 59.669 & 132.64 & 96.697 & 109.79 & 293.15 & 474.64 & 156.33 & 58.536 & 78.017 & 74.355 \\
\hline & 0.0431 & 0.42625 & 0.9475 & 0.69076 & 0.78426 & 2.09412 & 3.39063 & 1.11676 & 0.41815 & 0.55732 & 0.53116 \\
\hline \multirow[t]{2}{*}{${ }^{103} \mathrm{Rh}$} & 25.912 & 111.19 & 297.62 & 124.95 & 148.94 & 616.70 & 1094.4 & 249.09 & 27.319 & 41.583 & 26.426 \\
\hline & 0.10312 & 0.44248 & 1.18441 & 0.49725 & 0.59273 & 2.45422 & 4.35515 & 0.99128 & 0.10872 & 0.16548 & 0.10516 \\
\hline
\end{tabular}


TABLE V: $\chi_{n i}^{2}$ and $\chi_{n i}^{\overline{2}}$ for every nuclide

\begin{tabular}{|c|c|c|c|c|c|c|c|c|c|c|c|}
\hline Nucleus & $\begin{array}{l}\chi_{n G L}^{2} \\
\bar{\chi}_{n G L}^{2}\end{array}$ & $\begin{array}{l}\chi_{n C H 89}^{2} \\
\bar{\chi}_{n C H 89}^{2}\end{array}$ & $\begin{array}{l}\chi_{n K D}^{2} \\
\bar{\chi}_{n K D}^{2}\end{array}$ & $\begin{array}{l}\chi_{n S K a}^{2} \\
\bar{\chi}_{n S K a}^{2}\end{array}$ & $\begin{array}{l}\chi_{n S K b}^{2} \\
\bar{\chi}_{n S K b}^{2}\end{array}$ & $\begin{array}{c}\chi_{n G S 1}^{2} \\
\overline{\chi_{n G S 1}^{2}}\end{array}$ & $\begin{array}{c}\chi_{n G S 2}^{2} \\
\bar{\chi}_{n G S 2}^{2}\end{array}$ & $\begin{array}{l}\chi_{n G S 3}^{2} \\
\bar{\chi}_{n G S 3}^{2}\end{array}$ & $\begin{array}{l}\chi_{n G S 4}^{2} \\
\overline{\chi^{2}}{ }_{n G S 4}\end{array}$ & $\begin{array}{l}\chi_{n G S 5}^{2} \\
\bar{\chi}_{n G S 5}^{2}\end{array}$ & $\begin{array}{l}\chi_{n G S 6}^{2} \\
\bar{\chi}_{n G S 6}^{2}\end{array}$ \\
\hline \multirow[t]{2}{*}{${ }^{112} \mathrm{Sn}$} & 77.855 & 33.197 & 33.197 & 33.197 & 33.197 & 33.197 & 33.197 & 33.197 & 33.197 & 33.197 & 33.197 \\
\hline & 2.0897 & 0.89103 & 0.89103 & 0.89103 & 0.89103 & 0.89103 & 0.89103 & 0.89103 & 0.89103 & 0.89103 & 0.89103 \\
\hline \multirow[t]{2}{*}{${ }^{116} \mathrm{Sn}$} & 4.6477 & 313.44 & 67.688 & 12.586 & 13.315 & 34.442 & 27.398 & 20.246 & 628.64 & 88.560 & 40.459 \\
\hline & 0.04085 & 2.7551 & 0.59498 & 0.11063 & 0.11704 & 0.30274 & 0.24083 & 0.17796 & 5.52578 & 0.77844 & 0.35563 \\
\hline \multirow[t]{2}{*}{${ }^{118} \mathrm{Sn}$} & 9.4646 & 148.32 & 458.83 & 267.51 & 300.56 & 507.17 & 836.42 & 291.12 & 213.66 & 277.91 & 198.33 \\
\hline & 0.02967 & 0.46492 & 1.43822 & 0.83851 & 0.94212 & 1.58975 & 2.62179 & 0.91253 & 0.66972 & 0.8711 & 0.62167 \\
\hline \multirow[t]{2}{*}{${ }^{120} \mathrm{Sn}$} & 28.745 & 144.11 & 500.76 & 206.60 & 238.92 & 761.11 & 1240.7 & 324.49 & 162.90 & 195.20 & 163.12 \\
\hline & 0.07971 & 0.39962 & 1.38864 & 0.57293 & 0.66255 & 2.11064 & 3.44068 & 0.89984 & 0.45174 & 0.5413 & 0.45234 \\
\hline \multirow[t]{2}{*}{${ }^{124} \mathrm{Sn}$} & 13.999 & 41.086 & 81.178 & 86.983 & 100.78 & 130.79 & 180.37 & 75.561 & 50.654 & 76.368 & 59.802 \\
\hline & 0.17156 & 0.50352 & 0.99486 & 1.066 & 1.23513 & 1.6029 & 2.21043 & 0.92602 & 0.62078 & 0.93591 & 0.73289 \\
\hline \multirow[t]{2}{*}{${ }^{140} \mathrm{Ce}$} & 54.654 & 1990.5 & 3489.8 & 812.98 & 843.26 & 1101.4 & 1355.3 & 1267.8 & 922.97 & 958.50 & 1184.6 \\
\hline & 0.043 & 1.566 & 2.74556 & 0.63961 & 0.66343 & 0.8665 & 1.06627 & 0.99741 & 0.72615 & 0.7541 & 0.93197 \\
\hline \multirow[t]{2}{*}{${ }^{181} \mathrm{Ta}$} & 13.069 & 75.215 & 105.41 & 388.21 & 193.25 & 343.66 & 515.50 & 427.46 & 255.40 & 267.21 & 375.11 \\
\hline & 0.04857 & 0.27956 & 0.39179 & 1.44291 & 0.71826 & 1.27734 & 1.91603 & 1.58881 & 0.94928 & 0.9932 & 1.39424 \\
\hline \multirow[t]{2}{*}{${ }^{186} \mathrm{~W}$} & 1.0145 & 1244.7 & 1244.7 & 1244.7 & 1244.7 & 1244.7 & 1244.7 & 244.7 & 1244.7 & 1244.7 & 1244.7 \\
\hline & $8.96515 \mathrm{E}-4$ & 1.09991 & 1.09991 & 1.09991 & 1.09991 & 1.09991 & 1.09991 & 1.09991 & 1.09991 & 1.09991 & 1.09991 \\
\hline \multirow[t]{2}{*}{${ }^{197} \mathrm{Au}$} & 7.6812 & 35.696 & 75.052 & 87.911 & 106.42 & 247.08 & 445.72 & 135.90 & 59.359 & 69.881 & 72.441 \\
\hline & 0.06291 & 0.29234 & 0.61465 & 0.71996 & 0.87156 & 2.02355 & 3.65036 & 1.11295 & 0.48614 & 0.57231 & 0.59327 \\
\hline \multirow[t]{2}{*}{${ }^{206} \mathrm{~Pb}$} & 205.91 & 7309.3 & 7459.7 & 4733.7 & 4716.0 & 9804.2 & 9788.2 & 9489.0 & 8019.8 & 8002.3 & 8441.2 \\
\hline & 0.02905 & 1.0312 & 1.05242 & 0.66783 & 0.66534 & 1.38319 & 1.38094 & 1.33871 & 1.13144 & 1.12898 & 1.1909 \\
\hline \multirow[t]{2}{*}{${ }^{208} \mathrm{~Pb}$} & 15.849 & 80.832 & 113.44 & 144.37 & 161.28 & 297.72 & 437.82 & 235.44 & 152.40 & 163.79 & 182.23 \\
\hline & 0.08782 & 0.4479 & 0.6286 & 0.79994 & 0.89367 & 1.64971 & 2.42601 & 1.30456 & 0.84449 & 0.90757 & 1.00974 \\
\hline \multirow[t]{2}{*}{${ }^{209} \mathrm{Bi}$} & 101.66 & 178.16 & 184.73 & 124.73 & 124.26 & 148.18 & 4999.0 & 139.32 & 131.99 & 135.59 & 142.30 \\
\hline & 0.17446 & 0.30575 & 0.31702 & 0.21405 & 0.21325 & 0.25429 & 8.57874 & 0.23908 & 0.2265 & 0.23268 & 0.2442 \\
\hline \multirow[t]{2}{*}{${ }^{232} \mathrm{Th}$} & 35.618 & 69.459 & 100.16 & 371.41 & 410.92 & 526.24 & 508.48 & 468.44 & 364.68 & 417.11 & 437.94 \\
\hline & 0.10559 & 0.20592 & 0.29694 & 1.10109 & 1.2182 & 1.56009 & 1.50743 & 1.38873 & 1.08112 & 1.23657 & 1.29831 \\
\hline
\end{tabular}


TABLE VI: The values of $\overline{\chi_{i}^{2}},<\bar{\chi}^{2}{ }_{i}>$

\begin{tabular}{cccccccccccc}
\hline \hline $\mathrm{i}=$ & GL & CH89 & KD & SKa & SKb & GS1 & GS2 & GS3 & GS4 & GS5 & GS6 \\
\hline$\overline{\chi_{i}^{2}}$ & 51.1028 & 485.745 & 584.372 & 464.709 & 483.162 & 1152.13 & 1559.94 & 768.408 & 585.646 & 629.419 & 549.981 \\
$\overline{\chi^{2}}{ }_{i}>$ & 0.19786 & 0.79667 & 0.80986 & 0.67989 & 0.70624 & 1.61375 & 2.30155 & 1.05254 & 1.02148 & 1.0355 & 0.79799 \\
\hline \hline
\end{tabular}




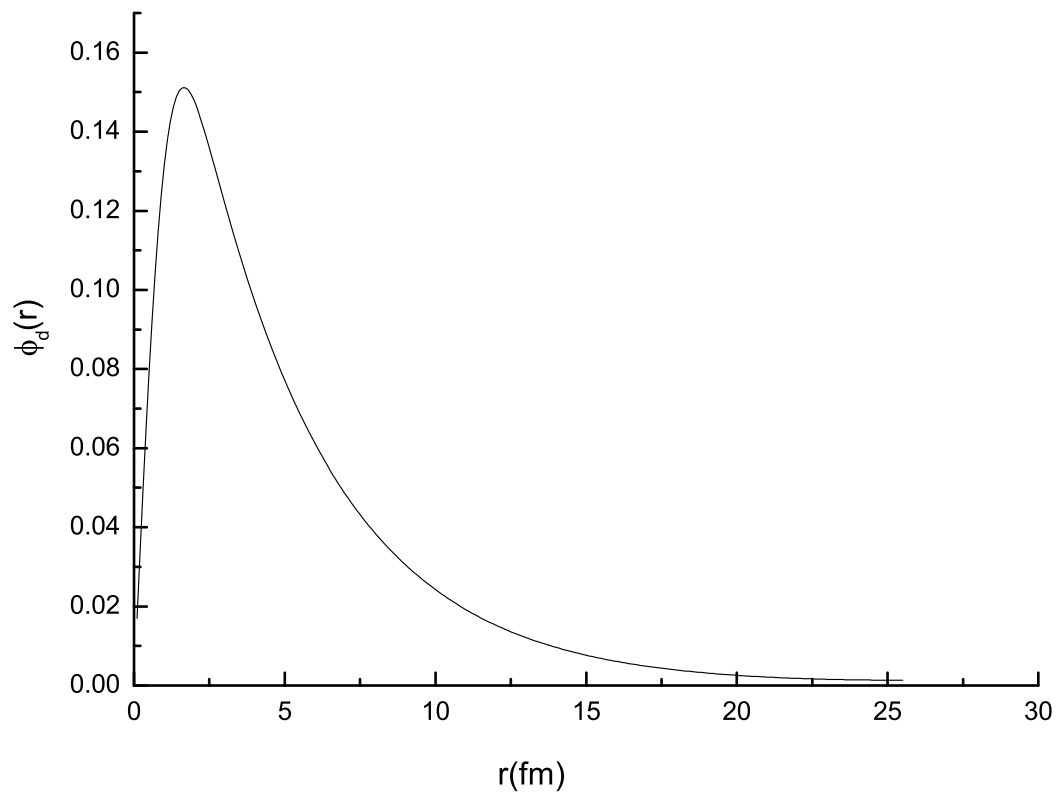

FIG. 1: The deuteron ground state wave function $\phi_{d}(r)$. 


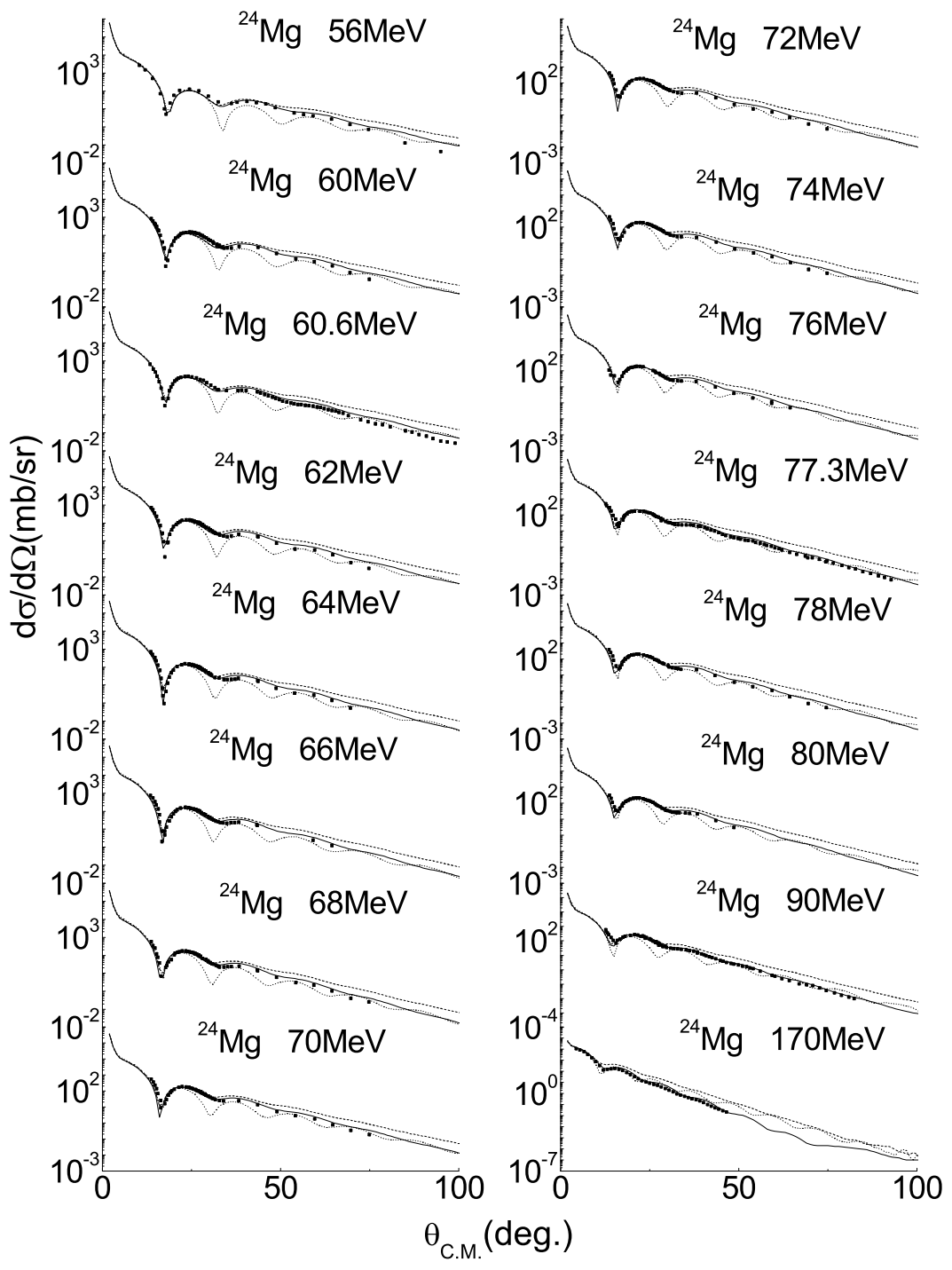

FIG. 2: Comparisons between the experimental angular distributions of elastic scattering in the center of mass frame for ${ }^{24} \mathrm{Mg}$ and the calculated values from the global deuteron optical potential parameters (GL) and the folding deuteron optical potential constructed with phenomenological global nucleon optical potential CH89 and the microscopic nucleon optical potential with the modified Skyrme force parameter SKa. The black dots denote the experimental data, the solid lines represent the values of GL, the dashed lines correspond to the values of CH89, the dotted lines to the values of SKa. The experimental data are taken from Refs. 19] [20] [21], the same symbols are used in other figures. 


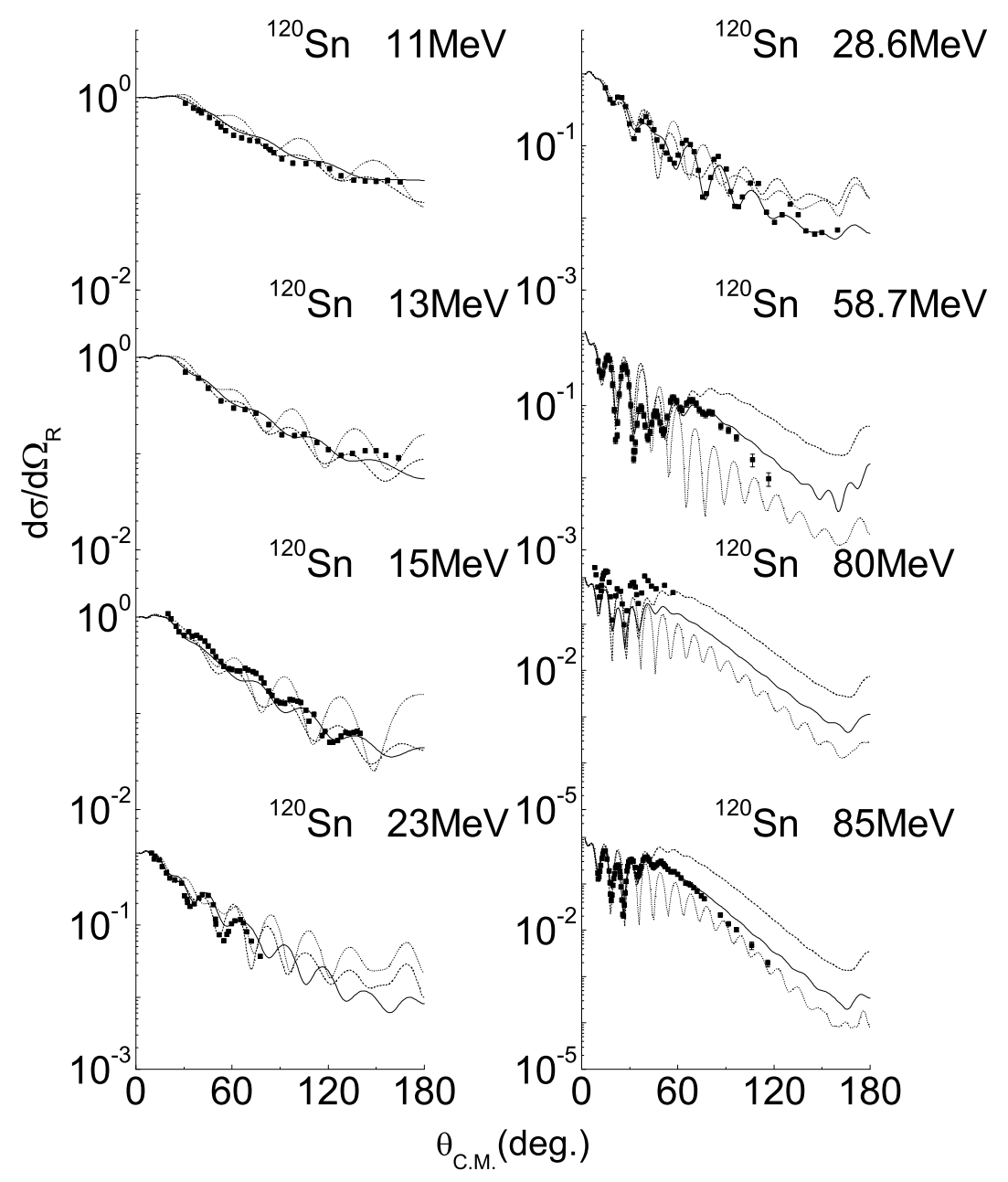

FIG. 3: Comparisons between the experimental angular distributions of elastic scattering in the center of mass frame for ${ }^{120} \mathrm{Sn}$ and the calculated values from the global deuteron optical potential parameters (GL) and the folding deuteron optical potential constructed with phenomenological global nucleon optical potential CH89 and the microscopic nucleon optical potential with the modified Skyrme force parameter SKa. The experimental data are taken from Ref. 22] [23] 24] [25] [26] [27]. 


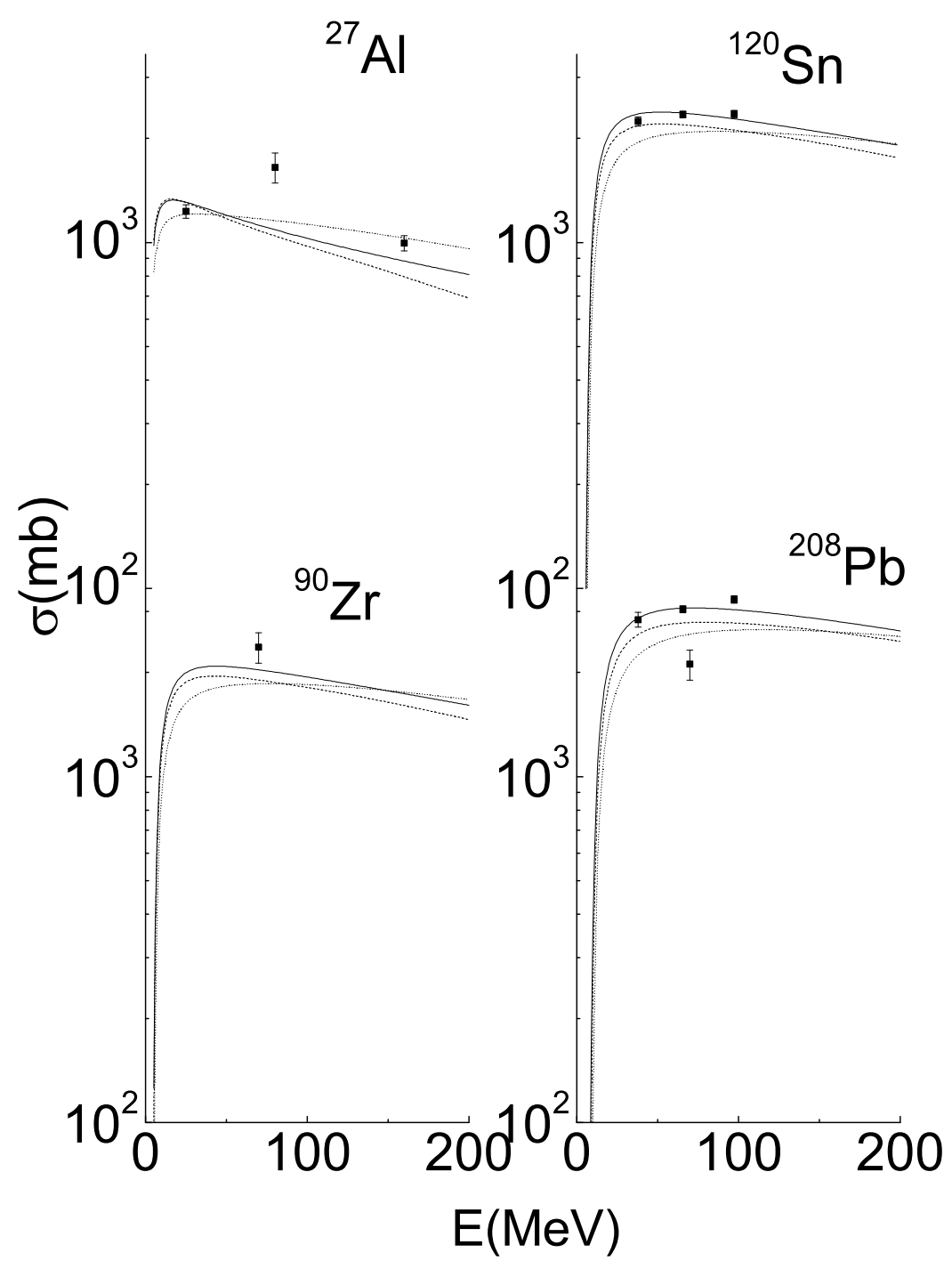

FIG. 4: Comparisons between the experimental reaction cross sections and the calculated values from the global deuteron optical potential parameters (GL) and the folding deuteron optical potential constructed with phenomenological global nucleon optical potential CH89 and the microscopic nucleon optical potential with the modified Skyrme force parameter SKa. The experimental data are taken from Ref. 28] 29] [30] 31]. 


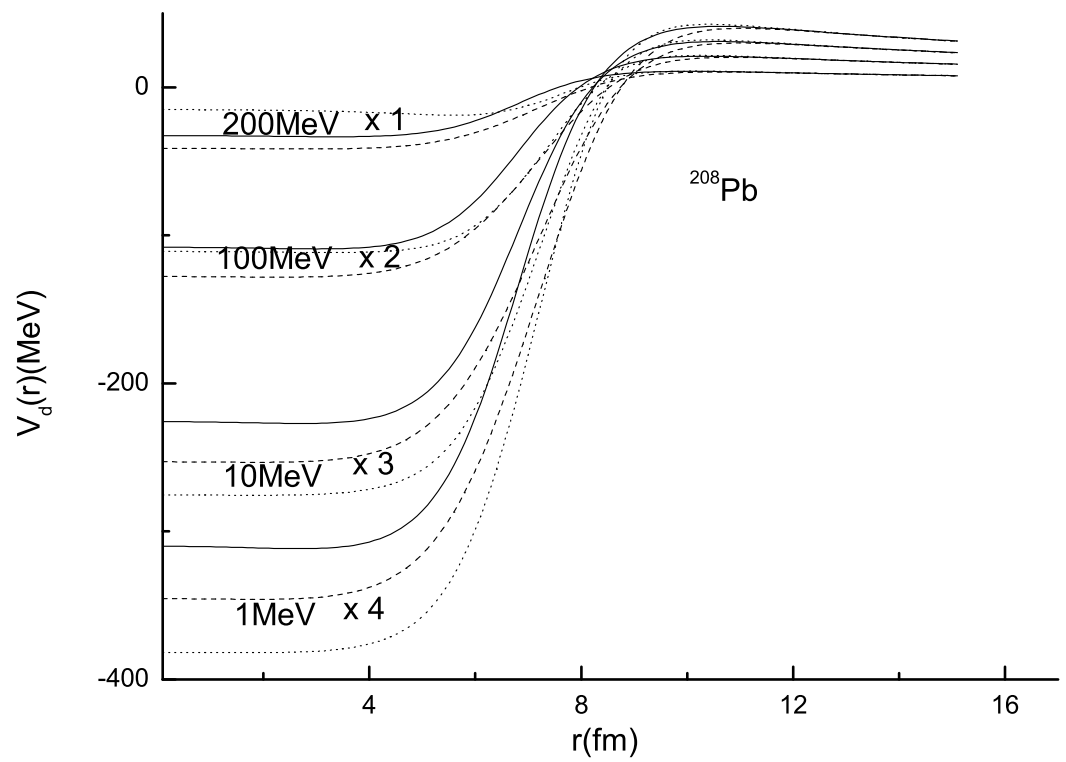

FIG. 5: Comparisons between the real parts of the folding deuteron optical potentials constructed with phenomenological global nucleon optical potential CH89 and the microscopic nucleon optical potential with the modified Skyrme force parameter SKa and those of the global deuteron optical potential for ${ }^{208} \mathrm{~Pb}$. 


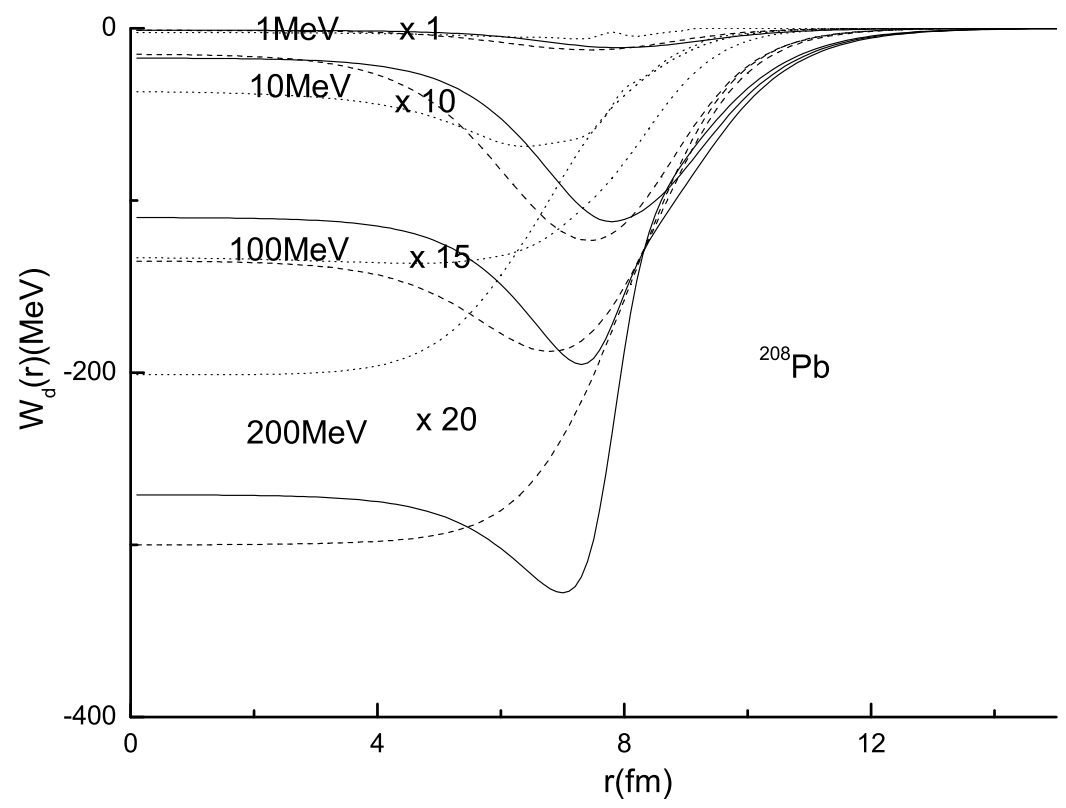

FIG. 6: Comparisons between the imaginary parts of the folding deuteron optical potentials constructed with phenomenological global nucleon optical potential CH89 and the microscopic nucleon optical potential with the modified Skyrme force parameter SKa and those of the global deuteron optical potential for ${ }^{208} \mathrm{~Pb}$. 